\title{
THE REPRESENTATION OF BOOLEAN ALGEBRAS
}

\author{
M. H. STONE
}

1. Introduction. In this brief address I shall set myself a twofold aim: to review the theory of Boolean algebras, as we understand it today, and to sketch certain historical phases of its development. Although I am in no sense prepared to offer the fruits of painstaking historical research, I believe that by a few pertinent historical observations I shall be able to bring out the underlying evolutionary pattern, which, in fact, is a quite familiar one.

2. Motivation and essential features of the theory. More than ninety years have passed since the publication of George Boole's first contribution towards an algebra of logic [5].* While Boole was by no means the first to attempt a symbolic method in logic (among his precursors we find Leibniz, Jacques and Jean Bernoulli, J. H. Lambert, and Gergonne [6]) it is a just and proper tribute to his genius that we commonly call this algebra by his name. I believe it would be accurate to say that of the many books, memoirs, notes, and reviews (more than one hundred seventy-five in number [6]) which deal with Boolean algebras the great majority draw their inspiration directly or indirectly from the work of Boole. The orientation of these studies toward symbolic logic is apparent in their preoccupation with algorithms, identities, and equations, or with the logical interrelations of the formal properties of the various Boolean operations. Recently there has emerged a different tendency, namely, to view Boolean algebras structurally, as organic systems, rather than algorithmically. Although this tendency might naturally have been expected to take its origin either in the rich experience of algebraists or in the needs of mathematicians concerned with the calculus of classes, it sprang, in fact, from quite different sources as a recognizable, if somewhat remote, consequence of the work of Hilbert. The most intensive exploitation of this new tendency is due to Tarski and myself [28]-[39]. Tarski's theory of deductive systems, which is but one illustration of the way in which logic has been enriched by the sort of metamathematical inquiry first seriously attempted by Hilbert, deals with systems of propositions which are complete with respect to logical inference; from a mathematical point of view, it is therefore a theory of the relations between special subalgebras of a

\footnotetext{
* References by number are to the bibliography at the end.
} 
Boolean algebra. My own investigations are a systematic attempt to discuss the structure of Boolean algebras by the methods which have thrown so much light on far deeper algebraic problems. The need for investigations of this character was suggested to me by the theory of operator-rings in Hilbert space: there, as in other rings and linear algebras, the "spectral" representation as a "direct sum" of irreduciible subrings reposes in essence upon the construction of an abstract Boolean algebra; and this construction, trivial for rings with strong chain conditions, is not trivial in the case of operator-rings.

Boolean algebras may be thought of as arising by abstraction from the familiar algebras of classes and point sets. The theory of Boolean algebras thus bears to the theory of combinations the same relation as the theory of abstract groups to the theory of permutations. Accordingly it is natural, even inevitable, that we should ask whether every abstract Boolean algebra corresponds isomorphically to some concrete algebra of classes or combinations, just as we ask whether every abstract group corresponds in like fashion to some concrete group of permutations. For the case of finite Boolean algebras, the answer has long been known ([12], p. 309) and is affirmative; the argument justifying it is almost as trivial as that which resolves the analogous question for all abstract groups. For the general case, the answer is not so easily obtained but is still affirmative. In fact, the theory of representations not only establishes the existence of an algebra of classes corresponding isomorphically to a given Boolean algebra, but even accomplishes the determination of all algebras of classes isomorphic or homomorphic to that algebra ([32], pp. 106111). On this occasion, I wish to emphasize especially the completeness of the theory of representations, inasmuch as a few reviews and abstracts of the theory [43] have not dwelt upon this feature. The essential mathematical steps in the development of the representation theory are easily summarized. They are first, the identification of Boolean algebras as Boolean rings with unit, where by the term "Boolean ring" is meant any ring in which every element is idempotent; ${ }^{*}$ second, the recognition of the representation problem as a special instance of the problem of imbedding a ring in a direct sum of rings of given type, and the consequent reduction of the problem to that of finding the prime ideals in a given Boolean ring; and third, the inductive construction of the desired prime ideals. I proceed to discuss these steps in greater detail.

* Boolean rings without unit correspond likewise to certain simple generalizations of Boolean algebras [31], [32]. 
3. Boolean algebras as rings. In the mathematical literature one frequently comes upon evidences of an opinion that Boolean algebras are in some sense fundamentally different from the algebras commonly met in dealing with families of real or complex numbers and their generalizations. Thus Whitehead [41] sets Boolean algebras apart as instances of "algebras of nonnumerical genus"; and quite recently Bell follows him, writing in the introductory remarks of a paper on Boolean algebras that "this is probably the first attempt to construct an arithmetic for an algebra of nonnumerical genus" [1].* The foundation for this opinion lies in the laws $a+a=a$ and $a a=a$ obeyed by the logical sum and product, and therefore vanishes unless one insists upon comparing these Boolean operations with arithmetic sum and product, respectively. Now there is a natural preference for these operations only when Boolean algebras are regarded as special instances of partially ordered sets or lattices; their "nonarithmetical" character is indeed sufficient reason for giving preference to others, if any more suitable ones can be found, when Boolean algebras are to be compared with common algebras. The fact is that, considered in terms of the appropriate Boolean operations, the Boolean algebras are precisely those Boolean rings which possess units [30], [32]: one may take the logical product as ring product, and the operation of forming the "symmetric difference" or "complete disjunction" as ring addition. In the algebra of classes, the product $A B$ is the class of elements common to $A$ and $B$, while the symmetric difference $A+B$ is the class of elements in $A$ or in $B$ but not both, familiar to topologists as the sum $(\bmod 2)$ of the classes $A$ and $B$. The essential formal properties of these preferred operations have long been known; they can be found scattered through the work of Schroeder [27], Daniell [7], Bernstein [2], Gégalkine [9], and presumably others. $\uparrow$ These properties suffice to show that every Boolean algebra is a Boolean ring with unit, a conclusion drawn explicitly but in slightly different terms by Orrin Frink in 1928 [8]. Frink showed further that, if the logical

* Indeed Bell, for reasons I do not understand, explicitly rejects a possibility which could have led him to the point of view described below; and Hurwitz [13] followed him in this respect. The correct determination of all congruences in a Boolean algebra I believe to be that given in [32], p. 81 .

$\dagger$ But Rinow's view [14] that Boole and C. S. Peirce were familiar with the properties of these operations is not adequately supported by his citations, it seems to me. Boole, for instance, rejects as simple a combination as $1+x$; and Peirce's "arithmetic sum" does not involve reduction $(\bmod 2)$. In fact, while Peirce obtains the ring properties for "arithmetic sum" and logical product, the resulting ring has the formal properties of the ring of functions (over an abstract set) assuming each a finite number of the values $0, \pm 1, \pm 2, \cdots$. 
product be taken a priori as the desired ring product, then the only Boolean operation which can be employed as ring addition is the operation of forming the symmetric difference. None of these authors, however, took the final and decisive step of showing that, conversely, every Boolean ring with unit is a Boolean algebra [30], [32].

In the present connection it is perhaps interesting to note a few simple properties of Boolean rings, and hence of Boolean algebras, in order to emphasize the relations with ordinary algebra. A Boolean ring is a domain of integrity (that is, is free from divisors of zero) if and only if it is isomorphic to the field $F_{2}$ of integers (mod 2) [2], [32].* Every Boolean ring satisfies the regularity condition of von Neumann [24]: the equation $a x a=a$ always has a solution, namely $x=a$. Finally, by virtue of the easily established law $2 a=a+a=0$, every Boolean ring is a linear associative algebra over the field $F_{2}$ [8].

4. Reduction of the representation problem. Only recently, McCoy and Montgomery [22] have stated in explicit terms the fundamental principle upon which the representation theory is based. It is this: A necessary and sufficient condition that a $\operatorname{ring} R$ be isomorphic to a subring of a direct sum (finite or infinite) of rings $K$ is that for every $a \neq 0$ in $R$ there exist a homomorphism $h$ of $R$ into a subring of $K$ with $h(a) \neq 0$. In order to appreciate the immediate relevance of this principle, let us observe that every Boolean ring of classes is isomorphic to a subring of a direct sum of the indicated kind. We have merely to recall the familiar reckoning with characteristic functions, as was done for similar purposes by $\mathrm{H}$. Whitney [42]. In a class $E$, the characteristic function $\phi_{A}$ of a subclass $A$, equal to 1 on $A$ and to 0 elsewhere, satisfies the following relations:

$$
\begin{aligned}
\phi_{A B} & =\phi_{A} \phi_{B}, \quad \phi_{A+B}=\phi_{A}+\phi_{B}(\bmod 2), \\
\phi_{A} & =0 \text { if and only if } A=O \text { (is void). }
\end{aligned}
$$

Evidently it is convenient to treat the values of $\phi_{A}$ as numbers of the field $F_{2}$. Now these relations show that the correspondence $A \rightarrow \phi_{A}$ carries the various Boolean rings of subclasses of $E$ isomorphically into Boolean rings of characteristic functions; in particular, it carries the ring of all subclasses of $E$ into the ring of all functions defined on $E$ with values in $F_{2}$, a ring which is precisely the direct sum of fields $F_{2}$ with one summand corresponding to each element of $E$. Accordingly, every Boolean ring of classes is isomorphic to a subring of a direct sum of fields $F_{2}$. The principle of McCoy and Montgomery therefore

* This field consists of two elements, 0 and 1 , with the rules of operation $0 \cdot 0=0 \cdot 1=1 \cdot 0=0+0=1+1=0$ and $1 \cdot 1=1+0=0+1=1$. 
reduces the representation problem for an abstract Boolean ring to the narrower problem of determining the homomorphisms of that ring into the field $F_{2}$. A simple algebraic discussion shows finally that the study of these homomorphisms is equivalent to the study of the prime ideals in the given ring ([32], pp. 85-86).

It is interesting to note that prior to the enunciation of the principle of McCoy and Montgomery a number of particular instances had appeared in the literature. Köthe [15], [16], and Prüfer [26] had used this principle without explicit statement, as I had in my own work on Boolean algebras. No doubt further instances could be found through a careful search of the literature. As McCoy and Montgomery point out, the principle may be more broadly formulated so as to apply to the most general kind of algebra and even to branches of mathematics outside algebra; for example, a similar criterion can be stated for the homeomorphism of a given topological space with a subspace of a prescribed Cartesian product. It is clear, I think, that McCoy and Montgomery are entitled to full credit for recognizing the great importance of this simple principle and for formulating it in general terms.

5. The construction of prime ideals. The representation theory for Boolean rings, as we have seen, rests ultimately upon the construction of prime ideals or, equivalently, of homomorphisms in to the field $F_{2}$. It is interesting and instructive to test the significance of the construction by reference to one or two concrete interpretations. In the case of an algebra of classes, the object of the construction is to assign to each class $A$ a real number $m(A)$ so that

$$
\begin{aligned}
m(A) & =0 \text { or } 1, \quad m(E)=1, \\
m(A B) & =m(A) m(B), \quad m(A+B)=m(A)+m(B)(\bmod 2),
\end{aligned}
$$

or, equivalently, so that

$$
\begin{aligned}
& m(A)=0 \text { or } 1, \quad m(E)=1, \quad m(O)=0, \\
& m(A \cup B)+m(A B)=m(A)+m(B) .
\end{aligned}
$$

In other words, we wish to construct a two-valued additive measure defined for the classes in the given Boolean algebra. In order to exclude trivial cases we may require further that for every one-element class $A$ the equation $m(A)=0$ be satisfied. In the case of an algebra of propositions, as envisaged by Boole and more fully developed by his successors, the object of the construction is to assign to each proposition $A$ a number $t(A)$ subject to requirements like those given above for $m$. It is easily verified that these requirements compel $t(A)$ to 
play the role of a truth-value. If we designate $A$ as true or false according as $t(A)=1$ or $t(A)=0$, the formal rules of logic are obeyed. These two interpretations strongly suggest that the desired construction can in general be effected only by an appeal to inductive methods, based on mathematical (or complete) induction in the case of countably infinite Boolean rings and on transfinite induction in the higher cases. Several inductive constructions of prime ideals in abstract Boolean rings have been given by various writers; all are comparatively simple and straightforward. I believe that the first of these constructions are the ones I obtained in the autumn of 1932 and later published ([28] and [32], pp. 100-104), though Garrett Birkhoff independently and at about the same time discovered a procedure which applies also to the case of distributive lattices ([3], pp. 458459). Further discussions have been given by von Neumann and Stone [25], and by McCoy and Montgomery [22].

It should be carefully observed, however, that certain of these constructions may be regarded as contained in somewhat earlier results established by Krull, Lindenbaum, Tarski, and Ulam. Ulam [40] and Tarski [36] independently constructed non-trivial solutions of the problem of measure indicated above; and their methods apply without essential modification to the theory of abstract Boolean rings. Lindenbaum [19] solved the analogous problem for truth-values in classical logic. In certain of his papers on the general theory of rings, Krull obtained constructions which are readily specialized to the case which concerns us here. For example, he proved the following result ([18], p. 732): In a commutative ring $R$ let $S$ be a multiplicative subsystem (that is, with $a$ and $b$ the system $S$ also contains $a b$ ) which does not contain 0 ; then there exists a prime ideal which does not contain $S$. The proof is exceedingly simple. By transfinite induction one easily constructs a "maximal" ideal not containing $S$, and one then shows, by an almost trivial argument, that this ideal must be prime. In the case of a Boolean ring we may take $S$ as consisting of a single element $a \neq 0$, since $a \cdot a=a$. Some of Krull's results on absolute value rings can also be specialized to yield the desired construction, as I have pointed out elsewhere [30].

6. Summary of the representation theory. The representation theory leads to the following results ([32], pp. 106-111). If \& is the class of all prime ideals in a Boolean ring $A$ and if $\mathbb{E}(a)$ is the class of all prime ideals not containing the element $a$, then the correspondence $a \rightarrow \&(a)$ defines an isomorphic representation of the ring $A$ (that is, $\mathbb{E}(a b)=\mathbb{E}(a) \mathbb{E}(b), \mathbb{E}(a+b)=\mathbb{E}(a)+\mathbb{E}(b)$, and $\mathbb{E}(a)$ is void if and only 
if $a=0$ ). Every algebra of classes homomorphic to $A$ can then be obtained from this representation by the suppression of fixed elements of $\&$ and the possible adjunction of superfluous elements. It is to be observed that the representation described here is a "maximal" one: the class $(5$ which is the substratum of the representation can be enlarged only by introducing superfluous elements ([32], pp. 106-111). Hence the following question is of some interest: How many elements of $\&$ must be retained in order that the algebra resulting from the suppression of all other elements be isomorphic to $A$ ? Since every Boolean algebra can now be regarded as an algebra of classes, we may rephrase the question in somewhat more general terms: If $A$ is a (reduced) algebra of subclasses of a class $E$ and $E$ is the class of all prime ideals in $A$, what relations hold between the cardinal numbers $|E|,|A|$, and $|\xi|$ ? For the infinite case simple estimates show that

$$
|E| \leqq|A| \leqq|\&|, \quad|A| \leqq 2^{|E|}, \quad|\xi| \leqq 2^{|A|} .
$$

Under the hypothesis that $\boldsymbol{N}_{\alpha+1}=2^{\boldsymbol{N}_{\alpha}}$, there are only four possibilities:

$$
\begin{aligned}
|E| & =|A|=|छ|, \\
|E| & =|A|<2^{|A|}=|\mathfrak{E}|, \\
|E|<2^{|E|} & =|A|=|\mathfrak{E}|, \\
|E|<2^{|E|} & =|A|<2^{|A|}=|\mathfrak{E}| .
\end{aligned}
$$

The fourth set of relations always holds in the finite case; but even in the simplest infinite case, where $|E|=\mathbf{N}_{0}$, all four sets of relations can be realized by proper choices of the algebra $A$.

7. Classification and ideal structure of Boolean rings. A problem which naturally arises in connection with the theory of Boolean rings is that of classification. In the finite case, a Boolean ring is completely determined by the number of its elements. In the infinite case, on the other hand, no such characterization in terms of simple invariants is possible. One can see this, for example, by noting the distinctions between algebras in which the formation of infinite unions or intersections is admitted with various qualifications. An abstract discussion of infinite unions leads at once to problems concerning ideal structure. If $\mathfrak{a}$ is a nonvoid subclass of a Boolean ring $A$, the union of its elements may be defined as the element $c$ for which the proposition " $x c=c$ " is equivalent to the proposition " $x a=a$ for every $a$ in $\mathfrak{a}$ "; or, equivalently, as the element $c$ for which the proposition " $c y=0$ " is equivalent to the proposition " $a y=0$ for every $a$ in $a$." If we denote 
by $\mathfrak{a}^{\prime}$ the class of all elements $y$ such that $a y=0$ for every $a$ in $\mathfrak{a}$, then $\mathfrak{a}^{\prime}$ is an ideal and $c$ is an element of the ideal $\mathfrak{a}^{\prime \prime}=\left(\mathfrak{a}^{\prime}\right)^{\prime} \supset \mathfrak{a}$. It is easily seen that $\mathfrak{a}^{\prime \prime \prime}=\left(\left(\mathfrak{a}^{\prime}\right)^{\prime}\right)^{\prime}=\mathfrak{a}^{\prime}$ and that, in consequence, $\mathfrak{a}$ and $\mathfrak{a}^{\prime \prime}$ have a common sum $c$. Thus to require the existence of $c$ is to require that the ideal $\mathfrak{a}^{\prime \prime}$ be principal (with $c$ as its generating element). We are thus led to study the algebra of ideals under the operations of forming ideal products and ideal sums and the operation ' introduced above, and to classify ideals according to their behavior under these operations (for example, I have called normal those ideals for which $\left.\mathfrak{a}=\mathfrak{a}^{\prime \prime}\right)$. An exhaustive study of this algebra [32], [34] permits us to distinguish a few special types of Boolean ring, but the results are, on the whole, meager. The most interesting are probably those which follow. It is possible to form arbitrary unions and intersections in a Boolean ring if and only if all its normal ideals are principal ([34], pp. 232-235). Any Boolean ring, by a simple construction on its normal ideals, can be imbedded in one for which arbitrary unions and intersections can be formed [20], [21], [32], [34]. A Boolean ring is isomorphic to the ring of all subclasses of some fixed class if and only if it admits the formation of arbitrary unions and intersections and, in addition, obeys the general form of the distributive law for infinite unions and intersections [34], [38].

The algebra of ideals in a Boolean ring has interesting relations to logic. In the classical logic of propositions, the ideals correspond to the deductive systems of Tarski [37], [39]. The theory outlined here thus includes the central features of a metamathematical discussion of deductive systems. On the other hand, the formal rules of the algebra are closely related to the Brouwer logic as elaborated by Heyting [11]. This connection was pointed out by Tarski ([39], p. 514) and by me ([32], p. 66). Certain aspects of this relationship are touched on in the contributions of Gödel [10], Kolmogoroff [17], and Tarski ([39], p. 514).

8. Topological considerations. A much deeper insight into the structure of Boolean rings is made possible by the introduction of topological concepts. A cardinal principle of modern mathematical research may be stated as a maxim: "One must always topologize." With this principle in mind, I attacked the representation $\mathfrak{a} \rightarrow \mathbb{E}(a)$ described above: each class $\mathbb{E}(a)$ was taken as a neighborhood of every one of its elements, the class $\&$ thus being converted into a topological space. It was found that $\&$ becomes a totally disconnected bicompact Hausdorff space with the sets $\mathbb{E}(a)$ as its open-and-closed sets; and that every totally disconnected bicompact Hausdorff space arises in precisely this way from a Boolean ring [29], [33]. The theory 
of Boolean rings is thus seen to be equivalent to the theory of a special kind of topological space. The difficulty of classifying infinite Boolean algebras now emerges in its proper light: even in the case of countably infinite Boolean algebras one is faced with the problem of finding invariants of zero-dimensional compact metric spaces (that is, closed subsets of the Cantor discontinuum). Although we lack a complete set of invariants in this much-studied case, we do have sufficient information to obtain a number of interesting results about deductive systems, as has recently been shown by Mostowski [23].

9. Distributive lattices. In closing, I wish to mention briefly the generalization from Boolean algebras to distributive lattices. While the methods and results of the theory of Boolean algebras can be extended, with suitable modifications, to the case of distributive lattices, the direct connection with the theory of rings is lost. For this reason I find that a result of MacNeille, proved at my suggestion, provides the most satisfactory approach to the theory of distributive lattices: every such lattice can be imbedded in an essentially unique "minimal" Boolean algebra by a purely algebraic construction [20], [21]. The relation between distributive lattices and Boolean rings is in this respect analogous to that between domains of integrity (commutative rings without divisors of zero) and fields. Nevertheless, the direct attack has many points of interest and should not be ignored in a systematic survey. In addition to MacNeille's discussion cited above, I may mention papers of Garrett Birkhoff [3], [4] and myself $[35]$.*

\section{BIBLIOGRAPHY}

1. E. T. Bell, Transactions of this Society, vol. 29 (1927), pp. 597-611.

2. B. A. Bernstein, Transactions of this Society, vol. 28 (1926), pp. 654-657.

3. Garrett Birkhoff, Proceedings of the Cambridge Philosophical Society, vol. 29 (1933), pp. 441-464.

4. —, Duke Mathematical Journal, vol. 3 (1937), pp. 443-454.

5. George Boole, The Mathematical Analysis of Logic, London and Cambridge, 1847, reprinted in Collected Works, vol. 1, Chicago and London, 1916.

6. Alonzo Church, $A$ bibliography of symbolic logic, Journal of Symbolic Logic, vol. 1 (1936), pp. 121-218.

7. P. J. Daniell, this Bulletin, vol. 23 (1916-1917), pp. 446-450.

8. Orrin Frink, this Bulletin, vol. 34 (1928), pp. 329-333.

9. J. J. Gégalkine, Matematicheskii Sbornik, vol. 35 (1928), pp. 311-373.

10. Kurt Gödel, Ergebnisse eines mathematischen Kolloquiums, vol. 4 (19311932), pp. 39-40.

* The historical remarks in Birkhoff's review of the latter in Zentralblatt [44] should, it seems to me, be completed by reference to the "reception dates" of the manuscripts of the two papers discussed there. 
11. A. Heyting, Sitzungsberichte der Preussischen Akademie der Wissenschaften, Physikalische-Mathematische Klasse (1930), pp. 42-56.

12. E. V. Huntington, Transactions of this Society, vol. 5 (1904), pp. 288-309.

13. W. A. Hurwitz, Transactions of this Society, vol. 30 (1928), pp. 420-424.

14. Jahrbuch über die Fortschritte der Mathematik, vol. 61 (1935), pp. 54-55.

15. Gottfried Köthe, Mathematische Annalen, vol. 103 (1930), pp. 545-572.

16. - - Nachrichten von der Gesellschaft der Wissenschaften zu Göttingen, Mathematisch-Physikalische Klasse (1930), pp. 195-207.

17. A. N. Kolmogoroff, Mathematische Zeitschrift, vol. 35 (1932), pp. 58-65.

18. W. Krull, Mathematische Annalen, vol. 101 (1929), pp. 729-744.

19. A. Lindenbaum, see [37], p. 394.

20. H. M. MacNeille, Proceedings of the National Academy of Sciences, vol. 22 (1936), pp. $45-50$.

21. - Transactions of this Society, vol. 42 (1937), pp. 416-460.

22. N. H. McCoy and Deane Montgomery, Duke Mathematical Journal, vol. 3 (1937), pp. 455-459.

23. A. Mostowski, Fundamenta Mathematicae, vol. 29 (1937), pp. 34-53.

24. J. von Neumann, Proceedings of the National Academy of Sciences, vol. 22 (1936), pp. 707-713.

25. J. von Neumann and M. H. Stone, Fundamenta Mathematicae, vol. 25 (1935), pp. 353-378.

26. H. Prüfer, Mathematische Annalen, vol. 94 (1925), pp. 198-243.

27. E. Schroeder, Algebra der Logik, $\mathrm{II}_{2}$, Leipzig, 1905, pp. 493-505.

28. M. H. Stone, this Bulletin, abstract 39-3-86.

29. - Proceedings of the National Academy of Sciences, vol. 20 (1934), pp. 197-202.

30. — - Proceedings of the National Academy of Sciences, vol. 21 (1935), pp. 103-105.

31. —-, American Journal of Mathematics, vol. 57 (1935), pp. 703-732.

32. —- Transactions of this Society, vol. 40 (1936), pp. 37-111.

33. —- Transactions of this Society, vol. 41 (1937), pp. 375-481.

34. —- Fundamenta Mathematicae, vol. 29 (1937), pp. 223-303.

35. - - Časopis pro Pěstování Matematiky a Fysiky, vol. 67 (1937), pp. $1-25$.

36. A. Tarski, Fundamenta Mathematicae, vol. 15 (1930), pp. 42-50. 404.

37. — , Monatshefte für Mathematik und Physik, vol. 37 (1930), pp. 361-

38. ——, Fundamenta Mathematicae, vol. 24 (1935), pp. 177-198.

39. - Fundamenta Mathematicae, vol. 25 (1935), pp. 503-526; vol. 26 (1936), pp. 283-301.

40. S. Ulam, Fundamenta Mathematicae, vol. 14 (1929), pp. 231-233.

41. A. N. Whitehead, Universal Algebra, vol. 1, Cambridge University Press, 1898 , p. 29.

42. H. Whitney, Annals of Mathematics, (2), vol. 34 (1933), pp. 405-414.

43. Zentralblatt für Mathematik, vol. 14 (1936), p. 340.

44. Zentralblatt für Mathematik, vol. 18 (1938), p. 3.

HARVARD UNIVERSITY 